\title{
Mechanism and free-energy barrier of the type-57 reconstruction of the zigzag edge of graphene
}

\author{
J. M. H. Kroes, ${ }^{1}$ M. A. Akhukov, ${ }^{1}$ J. H. Los, ${ }^{1}$ N. Pineau, ${ }^{2}$ and A. Fasolino ${ }^{1}$ \\ ${ }^{1}$ Radboud University Nijmegen/Institute for Molecules and Materials, Heyendaalseweg 135, NL-6525AJ Nijmegen, The Netherlands \\ ${ }^{2} C E A / D A M / D I F, F R-91297$ Arpajon, France
}

(Received 16 July 2010; revised manuscript received 4 February 2011; published 12 April 2011)

\begin{abstract}
Recent ab initio calculations without spin [P. Koskinen et al., Phys. Rev. Lett. 101, 115502 (2008)] predict that the zigzag edges of graphene should be reconstructed, albeit with an energy barrier to be overcome. After verifying that spin-polarized calculations give qualitatively the same result, we study the mechanism and the free energy of the reconstruction of the zigzag edges from a periodic hexagon structure (zz) to an alternate pentagon-heptagon structure $[\mathrm{zz}(57)]$ using the empirical long-range carbon bond order potential II (LCBOPII) potential. The zz(57) edges are stabilized by an almost triple bond similar to that of the armchair edges, and we propose a way to account for this quantum mechanical effect in the LCBOPII potential. Aside from that, the reconstructed edge is flat as a result of tensile edge stress. The reconstruction occurs spontaneously in molecular dynamics simulations at high temperature, leading to the identification of a reaction coordinate for the reconstruction that allows us to calculate the free-energy barrier by using Monte Carlo simulations and umbrella sampling. At room temperature, we find a free-energy barrier of $0.83 \mathrm{eV}$ for the first transformations of two hexagons to a pentagon-heptagon pair that is higher than the one for a fully reconstructed edge and increasing with temperature.
\end{abstract}

DOI: 10.1103/PhysRevB.83.165411

PACS number(s): 61.46.-w, 61.48.Gh

\section{INTRODUCTION}

The macroscopic properties of any material depend strongly upon the microscopic structure. In graphene nanoribbons (GNRs) ${ }^{1}$ (small stripes of graphene ${ }^{2}$ ), the edges play an important role because of the relatively large fraction of atoms situated at, or near, the edge. The band structure of GNRs differs substantially from that of bulk graphene and, depending on width ${ }^{3,4}$ and edge type (zigzag or armchair), it can be either metallic or semiconducting, $, 1,5,6$ the latter with great potential for applications in electronics. ${ }^{7,8}$ The zigzag termination is the most-studied edge in theoretical studies of transport in GNRs because it allows a simple definition of boundary conditions, decoupling the two sublattices of the hexagonal crystal. ${ }^{9}$ In these calculations, it is assumed that the atoms at the edges are saturated by hydrogen so that the band structure can be studied only in terms of the $\pi$ band, neglecting the lower $\sigma$ bands.

However, the situation might be more complicated than this. In fact, recent $a b$ initio calculations by Koskinen et al., ${ }^{10}$ based on the density functional theory (DFT), have found that the reconstruction of the zigzag edge (zz) to a structure with alternating pentagons and heptagons, hereafter called zz(57), leads to a lower edge energy and to a self-passivating edge that would not bind hydrogen atoms and may be identifiable through coherent electron focusing experiments. ${ }^{11}$ Aside from that, the authors predict the $\mathrm{zz}(57)$ structure to have an edge energy also slightly lower than that of the armchair (ac) edge (see Fig. 1 for the different structures). While the theoretical stability of this $\mathrm{zz}(57)$ edge is still a topic of debate, primarily due to the influence of hydrogen pressure in realistic systems, ${ }^{12-14}$ several recent transmission electron microscopy experiments ${ }^{15-17}$ have reported the experimental observation of a partially reconstructed zz edge. Furthermore, the semiconducting nature of this edge state may be a possible explanation as to why experimentally fabricated GNRs are predominantly semiconducting. ${ }^{18}$ Since it has been shown that the zz edges are magnetic, ${ }^{5,7}$ as a first step, we have verified, using DFT (Ref. 19) as implemented in the SIESTA code,${ }^{20}$ that the $\mathrm{zz}(57)$ edge is favored over $\mathrm{zz}$ and ac edges also when spin polarization is taken into account.

In this paper, we study the path and free-energy barriers for the zz to zz(57) edge reconstruction by means of Monte Carlo (MC) and molecular dynamics (MD) simulations based on the empirical potential long-range carbon bond order potential II (LCBOPII). ${ }^{21}$ The computational efficiency of this potential allows us to study not only the $T=0 \mathrm{~K}$ equilibrium structure of a fully reconstructed edge, but also to follow, for large samples and finite temperature, the path of reconstruction and to calculate the free-energy barrier for it. We find that the free-energy barrier for the reconstruction of a whole edge displays an unusual nonmonotonic trend, increasing up to about $700 \mathrm{~K}$ and slowly decreasing at higher temperatures. At room temperature, we find an energy barrier of $0.7 \mathrm{eV}$, in good agreement with the value of $0.6 \mathrm{eV}$ found with DFT at $T=0 \mathrm{~K} .{ }^{10}$ However, we find that the first step of the reconstruction, namely, the transformation of a single pair of hexagons into a pentagon-heptagon pair, has a higher freeenergy barrier of $0.83 \mathrm{eV}$ at room temperature, increasing to $\sim 1 \mathrm{eV}$ above $500 \mathrm{~K}$; therefore, it is this barrier that determines the escape rate. The reconstruction of this edge will most likely happen in a sequence of transformations since successive transformations next to already existing pentagon-heptagon pairs are significantly easier, as we will show.

To be able to describe correctly the edge reconstruction, we have slightly modified the bond order potential LCBOPII. According to DFT calculations, ${ }^{10}$ the $\mathrm{zz}(57)$ and ac edges have a strong, almost triple, bond at the edges that stabilizes these two structures with respect to the $\mathrm{zz}$ that has graphitic bonds. The strong character of this bond is not predicted by the original version of the LCBOPII potential. ${ }^{21}$ In fact, the conjugation term in the potential assumes that the electrons available for $\pi$-bond formation are equally shared among the bonds with undercoordinated neighbors. As a consequence, the edge bond (marked by a thick red line in Fig. 1) is assigned as a double bond, in disagreement with the DFT result. ${ }^{10}$ It is, 


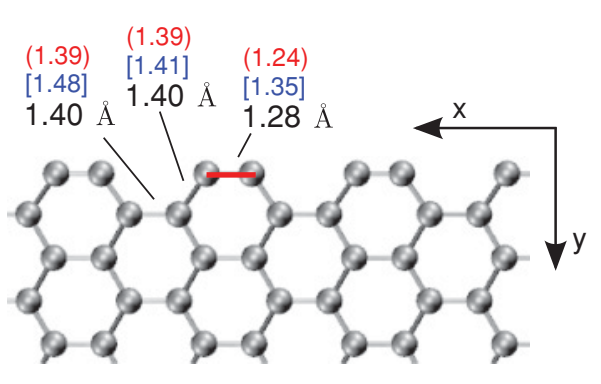

ac
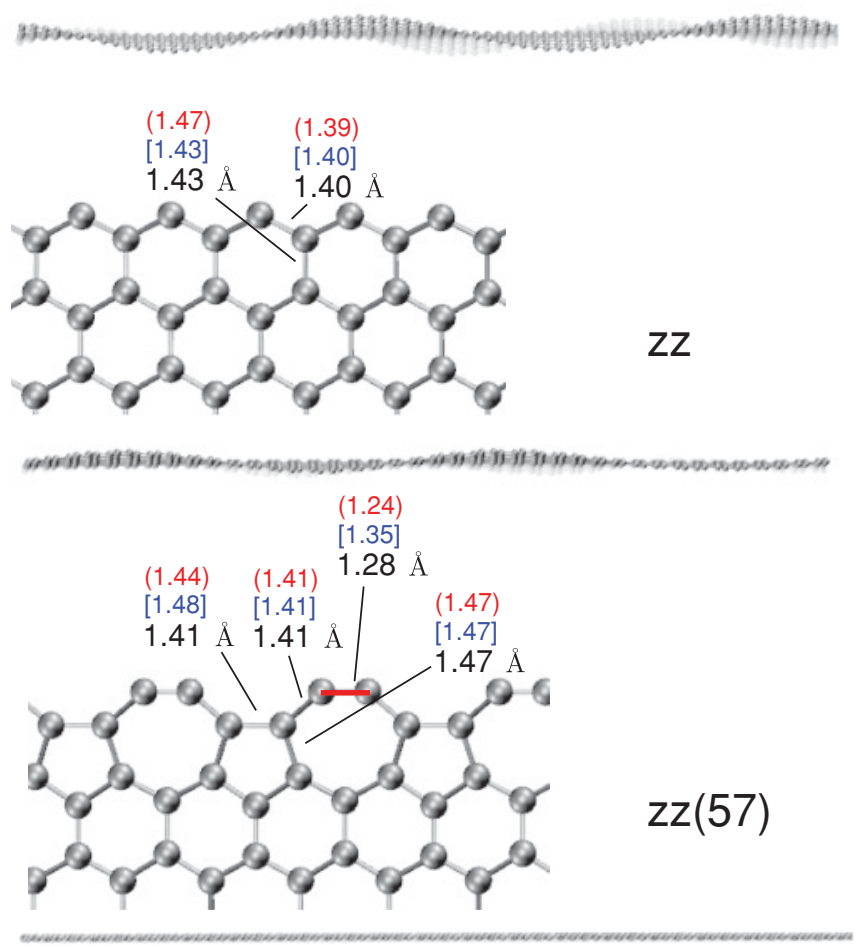

FIG. 1. (Color online) Top and lateral view of the equilibrium structure of (from top to bottom) ac, zz, and zz(57) edges obtained with the improved LCBOPII potential used in this paper with values of interatomic distances at the edge. For comparison, the DFT values (Ref. 10) are given in round parentheses and those given by the original LCBOPII (Ref. 21) are given in square brackets.

however, possible to modify the LCBOPII conjugation term in such a way that it gives the proper bond character without loss of computational efficiency. This is explained in detail in the Appendix.

The paper is organized as follows. In Sec. II, we show the structure and energetics at $T=0 \mathrm{~K}$ in comparison to the DFT results of Ref. 10 and to our own ab initio calculations done with the SIESTA package, ${ }^{20}$ the latter with and without taking into account spin polarization. In Sec. III, we derive a reaction coordinate for the transition from $\mathrm{zz}$ to $\mathrm{zz}(57)$ based on the results of MD simulations. In Sec. IV, we determine the free-energy barrier of the transformation of a full edge and of successive transformations of single pairs of hexagons to heptagon-pentagon pairs as a function of temperature. Section V contains the summary and conclusions.

\section{II. $T=0 \mathrm{~K}$ STRUCTURE AND ENERGETICS}

In Fig. 1, we show the zz, zz(57), and ac edge terminations. $A b$ initio DFT calculations ${ }^{10}$ predict that the most stable edge of graphene is the $z z(57)$, which can be considered as a reconstruction of the (metastable) zz edge. The $\mathrm{zz}(57)$ is also slightly favored over the ac edge.

Before addressing temperature-dependent properties, we have to verify that the empirical potential LCBOPII gives the correct behavior of graphene edges at $T=0 \mathrm{~K}$. The crucial feature is the armrest bond of $\mathrm{zz}(57)$, where, according to the DFT calculations of Ref. 10, the bond length decreases to $1.24 \AA$, a length slightly longer but comparable to that of a triple bond, which is $1.20 \AA$. This fact makes the bond selfpassivating because there are no more dangling bonds, as is the case for the $\mathrm{zz}$ edge. This feature is not reproduced by the original version of LCBOPII (Ref. 21) and, therefore, we have devised a simple modification described in the Appendix that allows us to capture this feature correctly. It should be noted that the situation at the $\mathrm{zz}(57)$ and ac edges, with a bond between two twofold-coordinated atoms, is not common. The modification used here and described in the Appendix changes only this situation, while keeping the description of all other bonding configurations equal, and, therefore, does not affect the zz edge.

Following Koskinen et al., ${ }^{10}$ we define the edge energy as

$$
\epsilon_{\text {edge }}=\frac{E-N \epsilon_{\text {bulk }}}{2 L},
$$

where $E$ is the total energy of the sample, $\epsilon_{\text {bulk }}$ is the bulk energy per particle, $L$ is the length of the edge, and the factor 2 accounts for the two edges of the sample. The energy difference between the $\mathrm{zz}$ and $\mathrm{zz}(57)$ is

$$
\Delta \epsilon=\left(\epsilon_{\text {edge }}^{\mathrm{zz}}-\epsilon_{\text {edge }}^{\mathrm{zz}(57)}\right) .
$$

For a detailed comparison between the results of LCBOPII with those obtained by DFT, we have performed DFT calculations of the edge energy and equilibrium structure by means of the package SIESTA, ${ }^{20}$ which implements DFT on a localized basis set. We used the generalized gradient approximation with Perdew-Burke-Ernzerhof parametrization (PBE-GGA) (Ref. 22) and did the calculations with and without taking into account spin polarization (SP), hereafter indicated as SP-SIESTA and SIESTA. A standard built-in double- $\zeta$ polarized (DZP) (Ref. 23) basis set was used for all calculations. The cutoff radii of the atomic orbitals were obtained from an energy shift equal to $1.0 \mathrm{mRy}$. The real-space grid is equivalent to a plane-wave cutoff energy of 360 Ry. For all nonperiodical directions, an extra space larger then $15 \AA$ was added to avoid spurious interactions. We used $50 k$ points for sampling the Brillouin zone. The geometry was relaxed using the conjugate gradient method until all forces were smaller than $0.02 \mathrm{eV} / \AA$. In agreement with Ref. 10, we find that, for samples with a periodically repeated minimal unit cell in the $x$ direction, the edge energy becomes constant for ribbon widths $\gtrsim 50 \AA$.

Next, we calculate the edge energy by MC simulations with LCBOPII for a sample $50 \AA$ wide, for which the edge energy is converged in the DFT calculations. In the $x$ direction, we take a periodically repeated cell of length $L_{x}=114 \AA$. We 
TABLE I. Edge energy $\epsilon_{\text {edge }}$ in $\mathrm{eV} / \AA$ obtained by (from left to right) the original LCBOPII (Ref. 21), LCBOPII with the modification described in the Appendix, the DFT package SIESTA (Ref. 20) without and with spin polarization, and by DFT in Ref. 10 without spin polarization. Note that the modified LCBOPII stabilizes also the ac edge that becomes slightly more favorable than the $\mathrm{zz}(57)$, at variance with the DFT results.

\begin{tabular}{lccccc}
\hline \hline & & Modified & & & \\
& LCBOPII & LCBOPII & SIESTA & SP-SIESTA & DFT \\
\hline $\mathrm{zz}$ & 1.05 & 1.05 & 1.34 & 1.15 & 1.31 \\
$\mathrm{ac}$ & 1.04 & 0.75 & 1.02 & 1.02 & 0.98 \\
$\mathrm{zz}(57)$ & 1.06 & 0.81 & 0.98 & 0.98 & 0.96 \\
\hline \hline
\end{tabular}

equilibrate the samples by performing MC simulations while lowering the temperature in steps from 100 to $0.1 \mathrm{~K}$.

In Table I, we compare the edge energies obtained by the different methods. Our DFT calculations with SIESTA give a value $\Delta \epsilon=0.33 \mathrm{eV} / \AA$ very close to the DFT value $\Delta \epsilon=$ $0.35 \mathrm{eV} / \AA ̊$ of Ref. 10. Including spin polarization in SIESTA gives the same qualitative result, favoring $\mathrm{zz}(57)$, although with a significantly smaller energy gain $\Delta \epsilon=0.13 \mathrm{eV} / \AA$.

In the original LCBOPII potential, ${ }^{21} \Delta \epsilon=-0.02 \mathrm{eV} / \AA$ (favoring the zz state). The modified potential (see Appendix) gives $\Delta \epsilon=0.24 \mathrm{eV} / \AA$ in qualitative agreement with the DFT results and in-between the values with and without $S P$.

In Fig. 1, we show the top and side views of the equilibrium edge structures and bond lengths found by DFT (Ref. 10) and by the original and modified LCBOPII. Note that the LCBOPII calculations allow out-of-plane relaxation that is neither included in Ref. 10 nor in our own DFT results due to the choice of a minimal cell in the periodic $x$ direction. The modified LCBOPII gives a bond length of the armrest of the $\operatorname{zz}(57)$ and ac edges of $1.28 \AA$, which is close to the

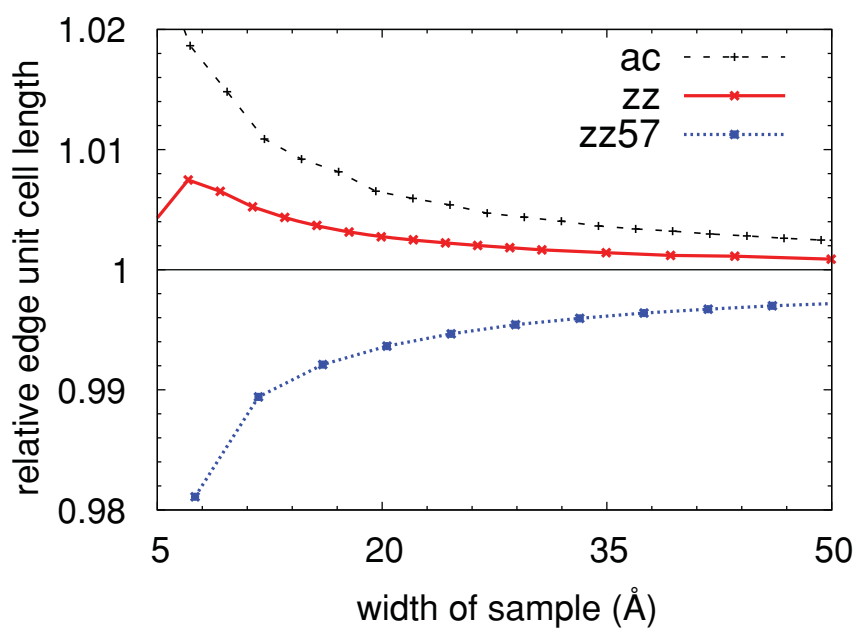

FIG. 2. (Color online) Equilibrium length in the periodic $x$ direction of the unit cell divided by the bulk value as a function of the width of the sample for ac, $\mathrm{zz}$, and $\mathrm{zz}(57)$ edges found using SIESTA. The zz and ac cells are larger than the bulk value (straight line) causing edge stress that would result in (out-of-plane) buckling of the edge. The $\mathrm{zz}(57)$ cell is smaller than the bulk and subjected to tensile stress favoring a flat geometry.
$1.24 \AA$ found by DFT. Furthermore, we note that the $\mathrm{zz}$ and ac edges are undulated in the out-of-plane direction, whereas the reconstructed $\mathrm{zz}(57)$ is completely flat. One possible reason is that the atoms at the edge favor a slightly different periodicity than the bulk one. Since the bulk size is fixed for wide enough samples, this will result in an edge stress. From Fig. 2, we can see that the equilibrium length of the unit cell in the periodic $x$ direction of GNRs with zz(57) edges calculated by SIESTA is slightly smaller than the bulk value, whereas for zz and ac, it is larger than the bulk value. As a consequence, $\mathrm{zz}(57)$ edges are subjected to tensile stress, while $\mathrm{zz}$ and ac are subjected to compressive stress that leads to out-of-plane distortion at the edges. The side views shown in Fig. 1 calculated by LCBOPII display exactly this behavior. These features match the description of the edge elastic properties described in Ref. 24.

\section{TRANSITION MECHANISM}

Next, we employed MD (Ref. 25) to examine the transition of the graphene edge from $\mathrm{zz}$ to $\mathrm{zz}(57)$. The MD simulation gives an insight into the dynamics of the transition. The simulation was done in the microcanonical (NVE) ensemble with time steps of 0.5 fs. The sample shown in the top panel of Fig. 3 is finite in all directions (no periodic boundary conditions). The temperature equilibrated to approximately $1500 \mathrm{~K}$, where the transition was found to take place within time scales of the order of picoseconds.

In Fig. 3, we show three snapshots during the transition of two hexagons at the zz edge to one pentagon-heptagon pair at the reconstructed edge. We see that, as the transition evolves, the distances $r_{A B}$ and $r_{A C}$ approximately interchange values, so that the nearest neighbors $A$ and $B$ become next nearest neighbors and the opposite for $A$ and $C$. Based on this finding, we define a reaction coordinate $d$ as

$$
d=\frac{r_{A B}-r_{57}}{r_{\mathrm{zZ}}-r_{57}},
$$

where $r_{\mathrm{zz}}=2.42 \AA$ and $r_{57}=1.47 \AA$ are the equilibrium values at $T=0 \mathrm{~K}$ of $r_{A B}$ for $\mathrm{zz}$ and $\mathrm{zz}(57)$, respectively. The identification of a reaction coordinate allows us to evaluate the free-energy barrier by umbrella sampling, as done in Sec. IV.

For reference, we first compared the (free-) energy barrier at $T=0 \mathrm{~K}$ according to LCBOPII with that according to SIESTA and SP-SIESTA for a reaction path obtained by linear interpolation (in 129 steps) of all atomic positions from the $\mathrm{zz}$ to the $\mathrm{zz}(57)$ configuration for a sample $16 \AA$ wide containing 32 atoms. The minimal energy configurations of the used initial zz-terminated and final zz(57)-terminated states were obtained with SIESTA. The energy barrier shown in Fig. 4, which does not include any further relaxation, represents an upper bound to the actual energy barrier. As a matter of fact, these values are about a factor 5 larger than those found with relaxation, as shown in Sec. IV, and are only meant to compare LCBOPII to SIESTA for a given fixed reaction path. The kink in the SP-SIESTA curve at $d \simeq 0.285$ marks the crossing of the energy curves from the non-SP and SP calculations, the one with the lowest energy being drawn here. The energy barrier according to LCBOPII is similar to that of SIESTA and SP-SIESTA, but LCBOPII gives a lower edge energy for both the $\mathrm{zz}$ and the $\mathrm{zz}(57)$. This fact 

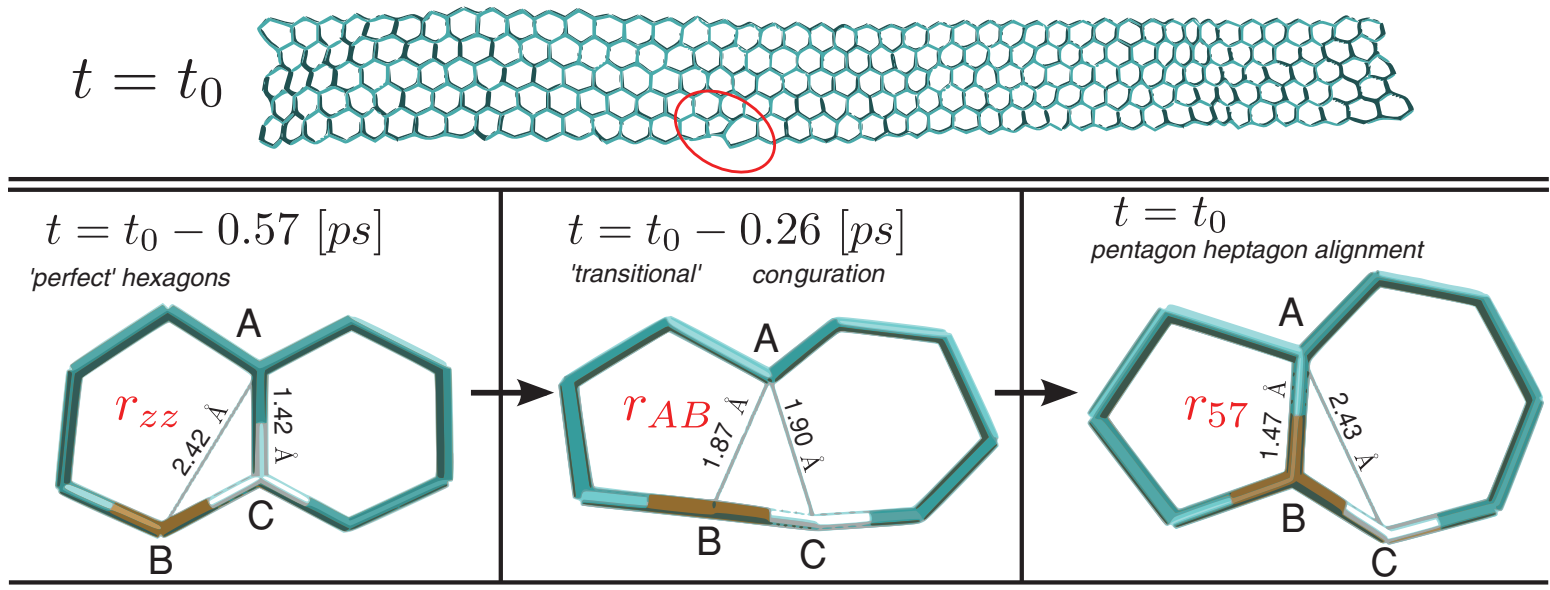

More transitions:
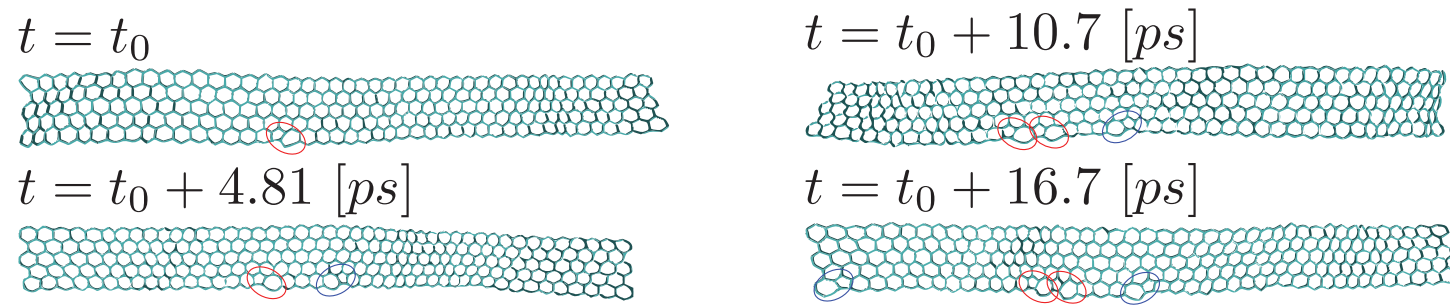$$
t=t_{0}+16.7[p s]
$$

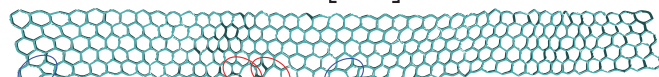

FIG. 3. (Color online) Top panel: Sample with one spontaneously reconstructed zz(57) cell. Middle panel: The first transition (at time $t=t_{0}$ ) is displayed in detail for different time frames, going from zz (upper left corner) to $\mathrm{zz}(57)$. This simulation was done at $\sim 1500 \mathrm{~K}$, in the NVE ensemble. Bottom panel: Successive transitions are shown where blue (right-oriented oval) and red represent the mirrored symmetric transitions.

can be partially explained by the too-high cohesive energies of the DFT methods. For example, SIESTA gives $7.90 \mathrm{eV}$ for bulk

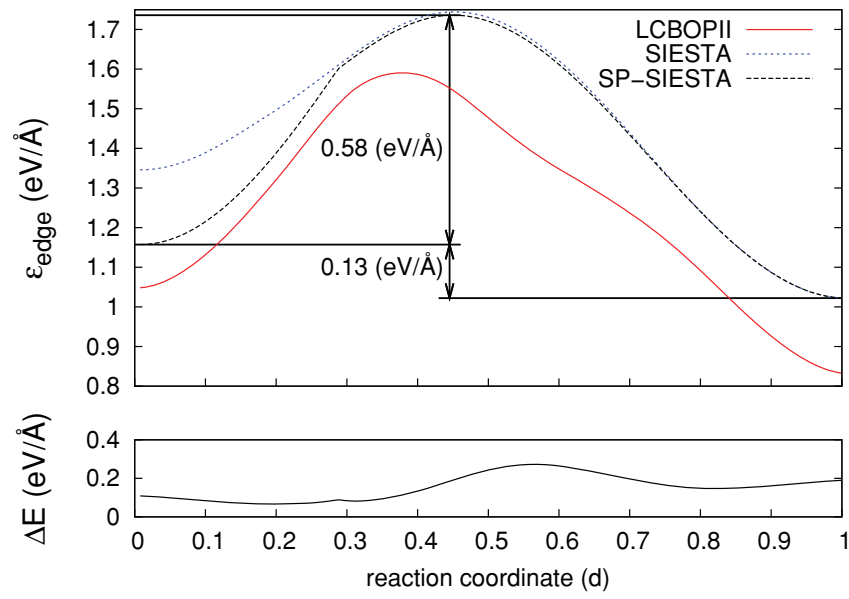

FIG. 4. (Color online) Top: Comparison between SIESTA(dotted blue line), SP-SIESTA (solid black line), and the modified LCBOPII (dashed red line) for the energy barrier from the $\mathrm{zz}$ state $(d=0)$ to the $\mathrm{zz}(57)$ state $(d=1)$ of a sample $16 \AA$ wide. The intermediate configurations are calculated by linearly interpolating the coordinates of the initial zz and final zz(57) state equilibrated in SP-SIESTA in 129 steps. This gives an upper bound to the energy barrier of $0.58 \mathrm{eV} / \AA$ $(0.52 \mathrm{eV} / \AA$ ) for SP-SIESTA (LCBOPII). Bottom: Difference $\Delta E$ between the energy barrier calculated by SP-SIESTA and the one calculated by the modified LCBOPII. graphene, against the experimental value of $7.35 \mathrm{eV}$ given by LCBOPII.

\section{FREE-ENERGY BARRIER}

In this section, we study the free-energy barrier for the $\mathrm{zz}$ to $\mathrm{zz}(57)$ transition, which requires us to study unit cells with two hexagons that transform into one pentagon-heptagon pair. Therefore, from now on, all energies are given per unit cell (4.94 $\AA$ ) rather than in $\mathrm{eV} / \AA$ as done previously.

The energy barrier between $\mathrm{zz}$ and $\mathrm{zz}(57)$ states, $\sim 0.6 \mathrm{eV}$ per edge unit cell according to Ref. 10, is extremely difficult to overcome by thermal fluctuations at low temperatures (below approximately $1500 \mathrm{~K}$ ) within computationally accessible time scales. An established technique to study this type of rare events computationally is the so-called umbrella sampling method (see, e.g., Ref. 26, p. 168). The method is illustrated in Fig. 5. We first divide the reaction coordinate interval $[0,1]$ into $n$ equal parts (called windows) labeled by $i \in\{1,2, \ldots, n\}$, where $n$ is chosen such that the maximum energy change in one window is of the order of the thermal energy. We then perform $n$ distinct MC simulations, where the modified LCBOPII potential $U_{\mathrm{LCBOPII}}$ is replaced by

$$
U= \begin{cases}U_{\text {LCBOPII }} & \text { if } \mathrm{d} \text { in window } \mathrm{i} \\ \infty & \text { else, }\end{cases}
$$

so that all MC moves outside the $i$ th umbrella window will be rejected. Second, by further dividing each window in 

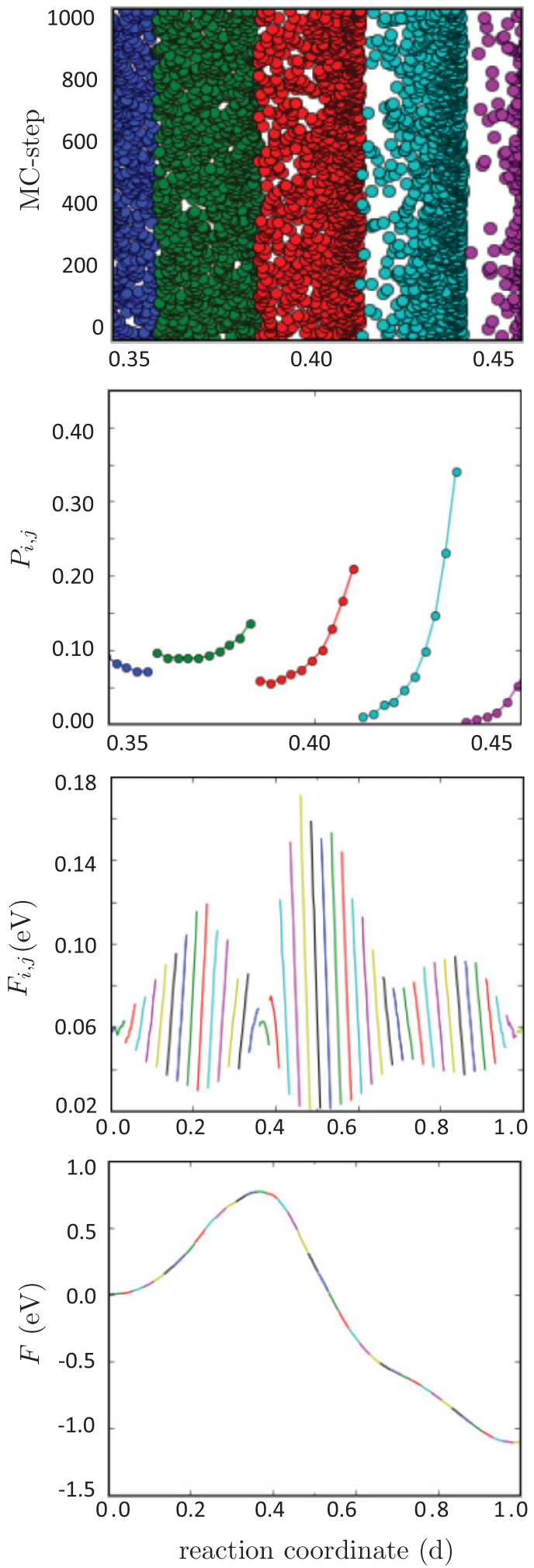

FIG. 5. (Color online) Four stages of umbrella sampling. From top to bottom: first 1000 sampling points for several windows 0.025 wide; probability $P_{i, j}$; relative free energy $F_{i, j}=-k T \log \left(P_{i, j}\right)$; free energy shifted by assuming continuity. Different colors are used to separate the windows.

$m$ subintervals (bins), we calculate the binned probability density $P_{i, j}$ for bin $j$ in window $i$, where $j \in\{0,1, \ldots, m\}$ (typically, $m=10$ ). Next, we can calculate the free energy up to an additive constant within each window for each bin as $F_{i, j}=-k_{\mathrm{B}} T \log \left(P_{i, j}\right)$, where $k_{\mathrm{B}}$ is the Boltzmann constant. By assuming that the free energy is continuous along the reaction path, the first $(n-1)$ additive constants can be found by linear extrapolation of the last two binned points to match the first point in the next window. The last additive constant is chosen to set the free energy of zz to zero.

In Fig. 6(a), we show the temperature dependence of the free energy along the transition path for a sample with two fully reconstructed edges. We find that the free-energy barrier $F_{b}=F_{\max }-F_{\mathrm{zz}}$ is nonmonotonic, growing up to $700 \mathrm{~K}$ and decreasing at higher temperature, as shown Fig. 6(b) The energy barrier at $T=0 \mathrm{~K}$, estimated by extrapolation, is approximately equal to $0.7 \mathrm{eV}$ per edge unit cell, close to the value $0.6 \mathrm{eV}$ obtained with the nudged elastic band method in Ref. 10. It is interesting to compare this behavior with that found for the initial step of the reconstruction, namely, the transformation of a single pair of hexagons into a pentagon-heptagon pair. The comparison to the one calculated for fully reconstructed edges in Fig. 6(b) shows that the free-energy barrier for the first transformation is definitely higher than that for a full reconstruction, it grows more rapidly with temperature, and does not decrease up to $1000 \mathrm{~K}$. This is almost the highest temperature we can study because, above the temperature where the transition occurs spontaneously, the umbrella sampling of single transformations can not be performed because transitions will occur spontaneously at other places.
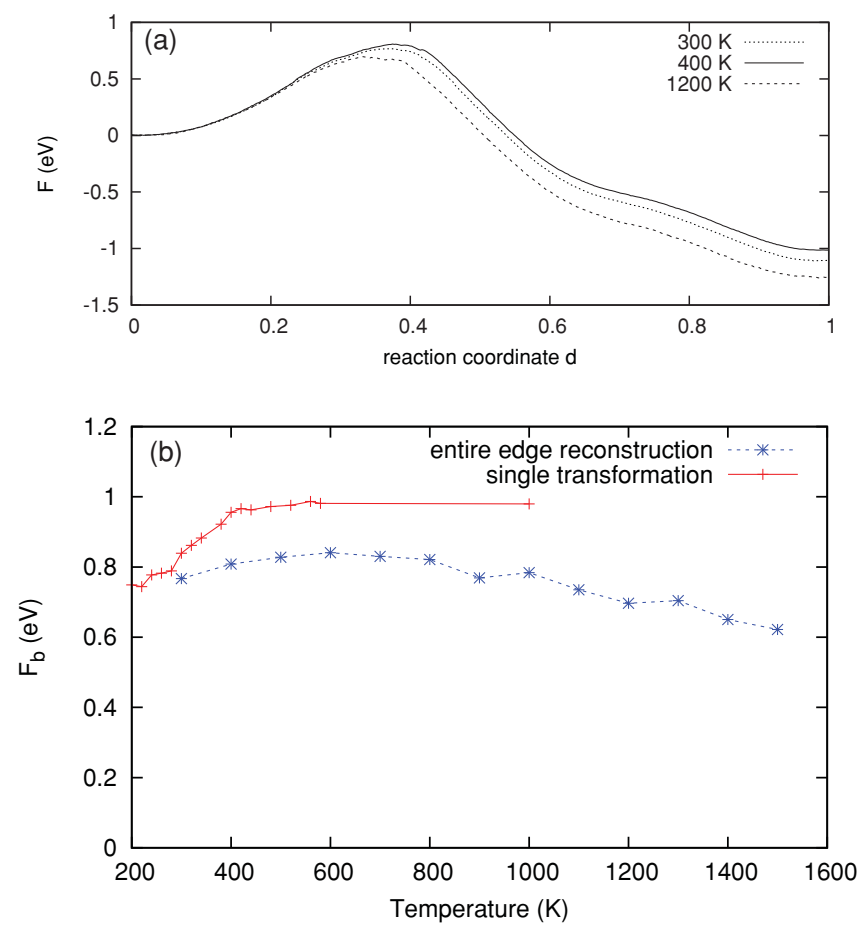

FIG. 6. (Color online) (a) Free energy per edge unit cell for different temperatures as a function of the reaction coordinate for the reconstructions of a whole edge. (b) Comparison of the temperature dependence of the free-energy barrier for the reconstruction of a whole edge $(*$, blue) with the free-energy barrier for the transformation of a single unit cell $(+$, red $)$. 


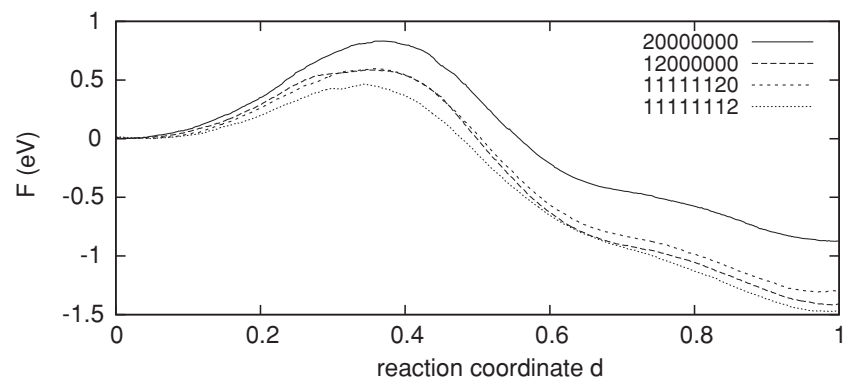

FIG. 7. Free energy per edge unit cell for successive transitions of a single pair of hexagons to a pentagon-heptagon pair as a function of the reaction coordinate. 0 means two zigzag cells at the edge, 1 means a (reconstructed) $\mathrm{zz}(57)$ cell, and 2 means the transition from $\mathrm{zz}$ to $\mathrm{zz}(57)$. The zero-energy point is chosen for convenience at the zz state.

Next we calculate the free-energy barrier for successive transformations of hexagon pairs. Our sample consists of $16 \mathrm{zz}$ edge unit cells (8 pairs of hexagons) in the periodic $x$ direction. To name different single transitions, we use an 8 -digit code consisting of the numbers 0 (two hexagons), 1 (pentagon-heptagon pair), and 2 (transition from 0 to 1 ). The free-energy barriers of the successive transitions at $T=300 \mathrm{~K}$ are shown in Fig. 7 (the same simulation was performed at $T=1000 \mathrm{~K})$. This shows that the free-energy barrier to create the first step of the reconstruction $(20000000)$ is the most difficult $\left[F_{b} \approx 0.83(0.98) \mathrm{eV}\right.$ at $\left.300(1000) \mathrm{K}\right]$. All following transitions $(12000000,11200000, \ldots)$ are progressively easier $\left[F_{b} \approx 0.71-0.59(0.79-0.69) \mathrm{eV}\right]$ and the last reconstruction (11 111 112) is the easiest [ $\left.F_{b} \approx 0.47(0.57) \mathrm{eV}\right]$.

Based on these results, we can now calculate a typical transition time for the first reconstruction (20000 000).

\section{A. Transition time}

For a single transition, we can use transition state theory ${ }^{27}$ to find a typical escape time $\tau_{e}$ for the system to go from one locally stable state $(A)$ to another $(B)$. In our case, the states represent, respectively, a zz sample (00 000000$)$ and the same sample with one heptagon-pentagon pair (10000000). The typical decay time from an energy minimum is given by

$$
\tau_{s} \sim M^{1 / 2}\left[\frac{d^{2} U}{d r^{2}}\right]_{r=r_{a}}^{-1 / 2},
$$

where $r_{a}$ is the equilibrium position of the system in state $A$ and $M$ is the mass of the particle escaping from the potential well that we take as the mass of a carbon atom. Fitting the free energy near the minimum at zz gives $\tau_{s} \approx 3.4 \times 10^{-14} \mathrm{~s}$. Then, an estimate of the time scale in which the system goes from $A$ to $B$, the escape time, is given by

$$
\tau_{e} \sim \tau_{s} \exp \left(F_{b} / E_{\text {noise }}\right) .
$$

We assume that $E_{\text {noise }}$ can be approximated by the thermal energy $E_{\text {noise }}=k_{\mathrm{B}} T$. At room temperature, with $F_{b}=0.83 \mathrm{eV}$, this gives a typical escape time of $\tau_{e} \approx 9 \mathrm{~s}$ decreasing to $\approx 2$ ps at $T=1000 \mathrm{~K}$ where $F_{b} \approx 1 \mathrm{eV}$. This will result in blurred images in electron microscopy because the edge can switch between the two states very fast, while, at low temperatures, the zz can be a stable edge. At $1500 \mathrm{~K}$, it can easily be observed in computer simulations and $\tau_{e}$ qualitatively matches the typical times between transitions as found in MD (see Fig. 3).

\section{CONCLUSION}

In summary, we have studied the edges of graphene nanoribbons using the LCBOPII potential by MD and MC simulations. A correct description of the armchair, and of the reconstructed $\mathrm{zz}(57)$, edge was achieved by a simple modification of the LCBOPII potential described in the Appendix. We have compared the equilibrium structure at $T=0 \mathrm{~K}$ with the results of $a b$ initio calculations ${ }^{10}$ and examined the role of out-of-plane displacements. We have identified a reaction coordinate for the $\mathrm{zz}$ to $\mathrm{zz}(57)$ edge reconstruction, which allows us to calculate, by umbrella sampling MC, the freeenergy profile for the transformation. At room temperature, we find the energy barrier for the reconstruction of a whole edge to be $0.7 \mathrm{eV}$ per edge unit cell, in good agreement with the value of $0.6 \mathrm{eV}$ found with DFT at $T=0 \mathrm{~K}$. However, we find that the first step of the reconstruction, namely, the transformation of a single pair of hexagons into a pentagon-heptagon pair, has a higher energy barrier of about $0.83 \mathrm{eV}$ and, therefore, it is this barrier that determines the escape rate. The escape time derived from this value might justify the abundance of nonreconstructed $\mathrm{zz}$ edges experimentally observed at room temperature.

\section{ACKNOWLEDGMENTS}

We thank Gilles de Wijs for discussions and Misha Katsnelson for his interest in this work. A.F. and M.A.A. acknowledge support from the Stichting voor Fundamenteel Onderzoek der Materie (FOM), which is financially supported by the Nederlandse Organisatie voor Wetenschappelijk Onderzoek (NWO).

\section{APPENDIX: IMPROVED TREATMENT OF CONJUGATION}

The hereafter described modification of LCBOPII to improve the description of the edge bonds of a graphene ribbon concerns the conjugation term $F_{i j}^{\text {conj }}$, which represents a contribution to the bond order. According to LCBOPII, ${ }^{21} F_{i j}^{\text {conj }}$ depends linearly on the number of valence electrons available for the bond $i j$ supplied by the atoms $i$ and $j$. In the original version, the four valence electrons of an undercoordinated $\mathrm{C}$ atom, i.e., an atom with less than four neighbors, are distributed according to the following rule. One electron is supplied to each bond with a saturated neighbor and the remaining electrons are equally shared among the bonds with the other neighbors. According to this rule, the graphene ac and $\mathrm{zz}(57)$ edge bonds [see Fig. 8(a)], with electron contributions of $N_{i j}^{e l}=N_{j i}^{e l}=2$ from atoms $i$ and $j$, will be assigned to be a double bond with an equilibrium bond distance of $1.33 \AA$. In contrast, DFT calculations predict an equilibrium bond distance of $1.24 \AA$, which corresponds to a bond strength in between double and triple, about $1 / 3$ of a double and $2 / 3$ of a triple bond. At the same time, according to the above LCBOPII rule, the contribution of $N_{i k}^{e l}=2$ electrons from atom $i$ to the 

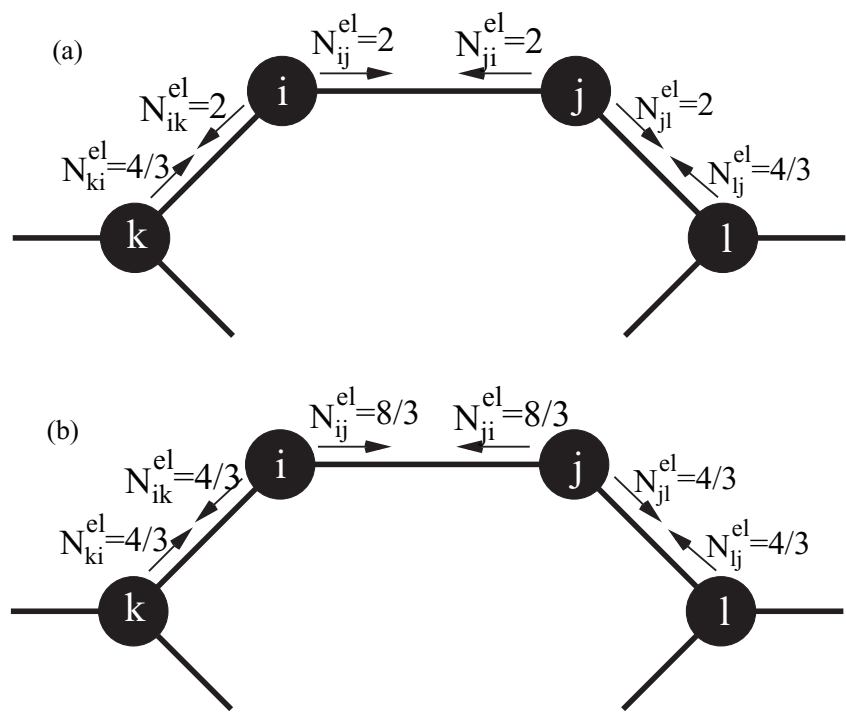

FIG. 8. Electron partitions according to (a) the original and (b) the modified LCBOPII.

bond $i k$ is not balanced by the contribution of $N_{k i}^{e l}=4 / 3$ electrons from the $\mathrm{sp}^{2}$ coordinated atom $k$, giving rise to a frustrated situation, which is penalized with the so-called antibonding term $A_{i j}$ in LCBOPII. This unfavorable situation can be resolved in a natural way by the alternative charge distribution shown in Fig. 8(b), in which the edge atom $i(j)$ supplies a number of electrons equal to $8 / 3=(1 / 3) \times 2=$ $(2 / 3) \times 3$ to the edge bond $i j$, leaving $4 / 3$ electrons of $i(j)$ for the bond $i k(j l)$, in balance with the electron supply $N_{k i}^{e l}\left(N_{l j}^{e l}\right)$ of the other neighbor $k(l)$.

To improve the electron partition rule of LCBOPII, one could think of minimizing the following functional:

$$
F\left(\left\{N_{i j}^{e l}\right\}\right)=\sum_{\langle i, j\rangle}\left(N_{i j}^{e l}-N_{i j}^{e l}\right)^{2},
$$

with the sum running over all neighbor pairs $i j$, under the constraints

$$
V_{i}=\sum_{i} N_{i j}^{e l}=4
$$

for all atoms $i$ in the systems, with $V_{i}=4$ being the (effective) number of valence electrons for carbon. It can be shown that, if one defines the electron charge on an atom $i$ as

$$
Q_{i}^{e l}=\frac{1}{2} \sum_{i}\left(N_{i j}^{e l}+N_{i j}^{e l}\right),
$$

the minimization of $F$ is equivalent to minimizing the charge transfer, providing a physical basis for our approach. However, minimizing $F$ in Eq. (A1) with the $N$ constraints (A2) is a nonlocal problem, which amounts to solving a coupled set of $\gamma N / 2-N$ linear equations, $\gamma$ being the average coordination number. For efficient MC or MD simulations, this is not desirable and we have to look for further approximations. To obtain the desired electron partition for the edge of a graphene ribbon, as depicted in Fig. 8(b), it appears to be sufficient to minimize only a part of the functional $F$, involving just the local environment of the edge bond $i j$. In particular, for bonds between two atoms with coordination 2, we minimize the functional

$$
\tilde{F}=\left(N_{i j}^{e l}-N_{j i}^{e l}\right)^{2}+W_{k i}\left(N_{i k}^{e l}-N_{k i}^{e l}\right)^{2}+W_{l j}\left(N_{j l}^{e l}-N_{l j}^{e l}\right)^{2},
$$

where the weight factors $W_{k i}$ and $W_{l j}$, defined below, are added to account for possible saturation of the atoms $k$ and $l$ and where $N_{k i}^{e l}$ and $N_{l j}^{e l}$ are fixed by the rule

$$
N_{k i}^{e l}=\max \left(1, \frac{V_{k}}{N_{k i}+1}\right)=\max \left(1, \frac{4}{N_{k i}+1}\right)
$$

and similarly for $N_{l j}^{e l}$. In Eq. (A5), $N_{k i}=N_{k}-S_{N, k i}^{\text {down }}$ is the socalled reduced coordination of atom $k, N_{k}=\sum_{m} S_{N, k m}^{\text {down }}$ being the total coordination of atom $k .^{21}$ Here, $S_{N}^{\text {down }}$ is a smooth cutoff function for the short-range (covalent) interactions, allowing for noninteger or fractional coordination. According to Eq. (A5), in the case of the ac and $\mathrm{zz}(57)$ graphene edges, $N_{k i}^{e l}=N_{l j}^{e l}=4 / 3$. Since, according to LCBOPII, the conjugation term in the case of a fractional neighbor $k(l)$ of atom $i(j)$ is evaluated as a weighted superposition of local configurations with only full neighbors (i.e., with $S_{N, i k}^{\text {down }}=1$ ), the denominator in Eq. (A5) is $N_{k i}+1$ instead of $N_{k i}+S_{N, k i}^{\text {down }}$. The weight factor $W_{k i}=W_{k i}\left(N_{k i}\right)$ in Eq. (A4) depends on $N_{k i}$, and is defined as

$$
W_{k i}=\frac{1}{1-S_{M, k i}^{\mathrm{up}}},
$$

where $S_{M, k i}^{\text {up }}$ is a switch function going from 0 to 1 for $N_{k i}+1$ going from 3 to 4 . Hence, $W_{k i}$ diverges when $k$ becomes a saturated neighbor, in which case minimization of $\tilde{F}$ will lead to $N_{i k}^{e l}=N_{k i}^{e l}=1$ as it should be. $W_{j l}$ is defined likewise. In Eq. (A4), $N_{i j}^{e l}$ and $N_{j i}^{e l}$ can be eliminated by using the constraints in Eq. (A2), leaving us to minimize $\tilde{F}$ with respect to the two variables $N_{i k}^{e l}$ and $N_{j l}^{e l}$, which, by straightforward minimization, leads to

$$
\begin{aligned}
N_{i k}^{e l} & =\frac{\left(2-S_{M, l j}^{\mathrm{up}}\right) N_{k i}^{e l}+\left(1-S_{M, k i}^{\mathrm{up}}\right) N_{l j}^{e l}}{3-S_{M, k i}^{\mathrm{up}}-S_{M, l j}^{\mathrm{up}}}, \\
N_{j l}^{e l} & =\frac{\left(1-S_{M, l j}^{\mathrm{up}}\right) N_{k i}^{e l}+\left(2-S_{M, k i}^{\mathrm{up}}\right) N_{l j}^{e l}}{3-S_{M, k i}^{\mathrm{up}}-S_{M, l j}^{\mathrm{up}}}
\end{aligned}
$$

from which $N_{i j}^{e l}$ and $N_{j i}^{e l}$ can be determined using Eq. (A2).

By applying Eqs. (A7) and (A8) to the graphene ribbon edge bond $i j$ in Fig. 8, with $S_{M, k i}^{\text {up }}=S_{M, l j}^{\text {up }}=0$, we find indeed the electron distribution given in Fig. 8(b). We note that the rule presented here correctly describes other configurations as well. For instance, if atom $k$ would have an additional neighbor, so that atom $k$ becomes saturated, then $S_{M, k i}^{\text {up }}=1, N_{k i}^{e l}=1$, and $N_{i k}^{e l}=1$ corresponding to a single bond. If also atom $l$ would 
have an additional neighbor, then we find $N_{j l}^{e l}=N_{i k}^{e l}=1$ and $N_{i j}^{e l}=N_{j i}^{e l}=3$ making $i j$ a triple bond as it should be. On the other hand, if both atoms $k$ and $l$ would have one neighbor less, then $S_{M, k i}^{\text {up }}=S_{M, l j}^{\text {up }}=0, N_{k i}^{e l}=N_{l j}^{e l}=2$, leading to a double bond with $N_{i j}^{e l}=N_{j i}^{e l}=2$.
For this paper, we have only modified the conjugation term for a bond between two twofold-coordinated atoms, i.e., atoms with reduced coordination $N_{i j}=N_{j i}=1$, where the effects are most significant. A more general treatment will be presented elsewhere. $^{28}$
${ }^{1}$ K. Nakada, M. Fujita, G. Dresselhaus, and M. S. Dresselhaus, Phys. Rev. B 54, 17954 (1996).

${ }^{2}$ K. S. Novoselov et al., Science 306, 666 (2004).

${ }^{3}$ M. Y. Han, B. Özyilmaz, Y. Zhang, and P. Kim, Phys. Rev. Lett. 98, 206805 (2007).

${ }^{4}$ Z. Chen, Y.-M. Lin, M. J. Rooks, and P. Avouris, Phys. E (Amsterdam) 40, 228 (2007).

${ }^{5}$ M. Fujita, K. Wakabayashi, K. Nakada, and K. Kusakabe, J. Phys. Soc. Jpn. 65, 1920 (1996).

${ }^{6}$ S. M.-M. Dubois, Z. Zanolli, X. Declerck, and J.-C. Charlier, Eur. Phys. J. B 72, 1 (2009).

${ }^{7}$ Y.-W. Son, M. L. Cohen, and S. G. Louie, Phys. Rev. Lett. 97, 216803 (2006).

${ }^{8}$ A. H. Castro Neto, F. Guinea, N. M. R. Peres, K. S. Novoselov, and A. K. Geim, Rev. Mod. Phys. 81, 109 (2009).

${ }^{9}$ L. Brey and H. A. Fertig, Phys. Rev. B 73, 235411 (2006).

${ }^{10}$ P. Koskinen, S. Malola, and H. Häkkinen, Phys. Rev. Lett. 101, 115502 (2008).

${ }^{11}$ P. Rakyta, A. Kormányos, J. Cserti, and P. Koskinen, Phys. Rev. B 81, 115411 (2010).

${ }^{12}$ T. Wassmann, A. P. Seitsonen, A. M. Saitta, M. Lazzeri, and F. Mauri, Phys. Rev. Lett. 101, 096402 (2008).

${ }^{13}$ T. Wassmann, A. P. Seitsonen, A. M. Saitta, M. Lazzeri, and F. Mauri, Phys. Status Solidi B 246, 2586 (2009).

${ }^{14}$ J. Kunstmann, C. Özdoğan, A. Quandt, and H. Fehske, Phys. Rev. B 83, 045414 (2011).
${ }^{15}$ P. Koskinen, S. Malola, and H. Häkkinen, Phys. Rev. B 80, 073401 (2009).

${ }^{16}$ Ç. Ö. Girit et al., Science 323, 1705 (2009).

${ }^{17}$ A. Chuvilin, J. C. Meyer, G. A-Siller, and U. Kaiser, New J. Phys. 11, 083019 (2009).

${ }^{18}$ J. Li, Z. Li, G. Zhou, Z. Liu, J. Wu, B.-L. Gu, J. Ihm, and W. Duan, Phys. Rev. B 82, 115410 (2010), and references therein.

${ }^{19}$ W. Kohn and L. J. Sham, Phys. Rev. 140, A1133 (1965).

${ }^{20}$ J. M. Soler, E. Artacho, J. D. Gale, A. Garcia, J. Junquera, P. Ordejon, and D. Sanchez-Portal, J. Phys. Condens. Matter 14, 2745 (2002).

${ }^{21}$ J. H. Los, L. M. Ghiringhelli, E. J. Meijer, and A. Fasolino, Phys. Rev. B 72, 214102 (2005).

${ }^{22}$ J. P. Perdew, K. Burke, and M. Ernzerhof, Phys. Rev. Lett. 77, 3865 (1996).

${ }^{23}$ J. Junquera, O. Paz, D. Sanchez-Portal, and E. Artacho, Phys. Rev. B 64, 235111 (2001).

${ }^{24}$ A. Cresti, N. Nemec, B. Biel, G. Niebler, F. Triozon, G. Cuniberti, and S. Roche, Nano Res. 1, 361 (2008).

${ }^{25} \mathrm{MD}$ simulations are performed with the parallel molecular dynamics code STAMP developed at CEA (France) using the original LCBOPII potential.

${ }^{26} \mathrm{D}$. Chandler, Introduction to Modern Statistical Mechanics (Oxford University Press, New York, 1987).

${ }^{27}$ P. Hänggi, P. Talkner, and M. Borkovec, Rev. Mod. Phys. 62, 253 (1990).

${ }^{28}$ J. H. Los et al. (unpublished). 